ISSN 2076-0817

www.mdpi.com/journal/pathogens

Review

\title{
Can We Prevent Antimicrobial Resistance by Using Antimicrobials Better?
}

\section{Germander Soothill ${ }^{1}$, Yanmin $\mathrm{Hu}^{2}$ and Anthony Coates ${ }^{2, *}$}

1 Barts and The London School of Medicine and Dentistry, Queen Mary, University of London, Garrod Building, Turner Street, Whitechapel, London, E1 2AD, UK;

E-Mail: g.soothill@smd10.qmul.ac.uk

2 Medical Microbiology, Centre for Infection, Division of Clinical Studies, St George's, University of London, Cranmer Terrace, London SW17 0RE, UK; E-Mail: ymhu@ sgul.ac.uk

* Author to whom correspondence should be addressed; E-Mail: acoates@ sgul.ac.uk.

Received: 23 April 2013; in revised form: 24 May 2013 / Accepted: 1 June 2013 /

Published: 10 June 2013

\begin{abstract}
Since their development over 60 years ago, antimicrobials have become an integral part of healthcare practice worldwide. Recently, this has been put in jeopardy by the emergence of widespread antimicrobial resistance, which is one of the major problems facing modern medicine. In the past, the development of new antimicrobials kept us one step ahead of the problem of resistance, but only three new classes of antimicrobials have reached the market in the last thirty years. A time is therefore approaching when we may not have effective treatment against bacterial infections, particularly for those that are caused by Gram-negative organisms. An important strategy to reduce the development of antimicrobial resistance is to use antimicrobials more appropriately, in ways that will prevent resistance. This involves a consideration of the pharmacokinetic and pharmacodynamics properties of antimicrobials, the possible use of combinations, and more appropriate choice of antimicrobials, which may include rapid diagnostic testing and antimicrobial cycling. Examples given in this review include Mycobacterium tuberculosis, Gram-negative and Gram-positive organisms. We shall summarise the current evidence for these strategies and outline areas for future development.
\end{abstract}


Keywords: antimicrobial resistance; pharmacokinetics; pharmacodynamics; minimum inhibitory concentration (MIC); mutant prevention concentration (MPC); microbiota; colonisation resistance; antibiotic combination; bacteria-antimicrobial pairing; antimicrobial cycling

\section{Introduction: The Problem of Antimicrobial Resistance}

The theme of World Health Day 2011 "antimicrobial resistance: no action today, no cure tomorrow" highlighted antimicrobial resistance as a major issue. Boucher et al. describe the pathogens currently presenting the biggest problem in terms of antimicrobial resistance as the ESKAPE pathogens: Enterococcus faecium, Staphylococcus aureus, Klebsiella pneumoniae, Acinetobacter baumanii, Pseudomonas aeruginosa, and Enterobacter species [1,2]. The detection of the Metallobeta-lactamase (NDM-1) enzyme in 2008 alarmed the international community [3,4]. There is a lack of treatment options to deal with some of the multidrug resistant pathogens. The result is the use of (i) last-line therapies such as carbapenems, that would ideally be preserved [5]; and (ii) antimicrobials previously discarded due to toxicity, such as polymyxins, which have been brought back into use despite nephrotoxicity and neurotoxicity [6].

The development of novel antimicrobials is a subject of considerable interest, which has been discussed in depth by commentators [7-12]. However, new drug development alone cannot solve the problem. With few new classes of antimicrobials currently in the pipeline, and others that are still years away from the market, resistance prevention strategies have a crucial role to play in preserving the lifespan of existing antimicrobials $[13,14]$.

Strategies to extend the lifespan of the antimicrobials by preventing antimicrobial resistance include: (i) reducing usage of antimicrobials; (ii) appropriate use of antimicrobials; and (iii) infection control [15]. According to the World Health Organisation, limiting the development of antimicrobial resistance ultimately lies with reducing antimicrobial usage [16]. Reducing antimicrobial usage requires tackling a number of difficult areas, including reducing over-the counter purchases, public health campaigns, education of medical professionals, and reducing antimicrobial usage in animals, as has been discussed by excellent reviews on the subject [5,17-21]. Preventing cross-infection with resistant bacteria prevents the spread of antimicrobial resistant infections and so infection control is an important strategy, including outbreak control, hand hygiene, barrier precautions, and prophylactic antimicrobials, as discussed by reviews [13,16,22,23]. This review will focus on the second core strategy, to extend the lifespan of current antimicrobials by using the ones we have in ways that will reduce the emergence of resistance.

\section{Appropriate Antimicrobial Usage}

Clinically relevant resistance due to chromosomal mutations develops gradually due to the accumulation of alterations [24]. The mutation rate differs between antimicrobial-bacteria pairings because some antimicrobials select resistant mutations in certain bacteria more readily. Choice of antimicrobial-bacterial pairing, antimicrobial combinations, and appropriate dosing can potentially reduce 
the emergence of de novo resistance. A good example of this is treatment of Mycobacterium tuberculosis, in which resistance arises solely by chromosomal mutations (the successful use of combination therapy to avoid resistant mutants for Mycobacterium tuberculosis is discussed in section 2.2.). In contrast, the transfer of resistance genes, for instance via plasmids, can confer clinically relevant resistance in a single step. Slowing the progress of this type of resistance is extremely difficult [24]. This type of resistance transfer is particularly common in multi-species populations in the large intestine in humans. Horizontal gene-transfer accounts for much of resistance to antimicrobials in hospital-acquired infections for the majority of antimicrobial classes [25].

\subsection{Pharmacokinetics and Pharmacodynamics}

Pharmacokinetics and pharmacodynamics are topics of great importance in the areas of antibacterial efficacy and clinical outcomes [26]; recently they have also been applied to the topic of antimicrobial resistance.

\subsubsection{Dosage}

Progress in the understanding of how the dose of antimicrobials affects resistance has been reviewed by Olofsson et al [27]. They describe how single high doses might be better at preventing resistance than multiple low doses (as shown in both in vitro and in vivo models [28-34]). Further research is required to highlight the optimal doses, which will successfully treat bacterial infections while preventing resistance.

Consensus has not yet been reached regarding which parameter of dose assessment should be used in resistance studies. Minimum inhibitory concentration (MIC) is the minimum concentration of an antimicrobial that will inhibit the growth of bacteria after an appropriate period of incubation [26]. Blaser et al. have showed that maximising the ratio of maximum concentration in serum $\left(\mathrm{C}_{\max }\right)$ to $\mathrm{MIC}$ restricted the growth of resistant mutants in in vitro studies [28]. Moreover, Thomas et al. have suggested that selection of antimicrobial resistance is decreased if the ratio of the area under the curve (AUC) to MIC is greater than 100 [35]. Traditionally, the efficacy of anti-tuberculosis antimicrobials has been associated with a high AUC to MIC ratio. However, in contrast, it has recently been shown that in the case of Mycobacterium tuberculosis treatment, the efficacy of isoniazid and rifamycins may be determined by peak drug concentrations [36]. More research in this area is needed but, if this concept is confirmed, then high doses of rifamycins may be able to shorten tuberculosis treatment. It is anticipated that shortening the duration of tuberculosis chemotherapy should lead to an increase in patient adherence [37,38].

Blondeau et al. examined the effect of using high dose fluroquinolones against Streptococcus pneumoniae in vitro studies [39]. They used concentrations of fluroquinolones equal to the mutation prevention concentration (MPC), concentration above which no first step resistant mutants grow, in order to slow the development of fluroquinolone resistance. In vivo studies in rabbits show that the use of a levofloxacin concentration greater than MPC supresses the growth of mutant populations in Staphylococcus aureus, and use of a vancomycin concentration above MPC or with a high AUC to MIC ratio reduces the acquisition of resistance in methicillin-resistant Staphylococcus aureus [40,41]. Whether dosing to achieve the MPC will prevent resistance development in human patients needs 
more investigation. Since each antimicrobial-bacterial pairing has a unique MPC, it may not always be possible to achieve the MPC with a safe antimicrobial dose [42]. Recent clinical trial data with linezolid show efficacy in extensively drug-resistant tuberculosis, however $82 \%$ of patients experienced clinically significant adverse events [43]. Although it is bacteriostatic in vitro, it reaches high concentrations in the lungs, which somewhat blurs bacteriostatic/bactericidal relevance in vivo. This also alludes to the potential role of MPC in reducing resistance. More research is needed to ascertain which parameter and levels of dose are optimal to prevent antimicrobial resistance $[44,45]$.

Mathematical models are being used to examine the theoretical basis for shorter treatment courses and optimise the duration and dosing of antimicrobials, which will minimise the duration of symptomatic infection as well as the selection pressure for resistance. Recent conclusions from these studies are that long durations of antimicrobial therapy are less appropriate for self-limiting infections and that the potential for selection for resistance in commensal flora should be taken into account when choosing the duration of therapy [46].

\subsubsection{Distribution and Excretion}

The site of infection and infecting bacteria are not the only considerations for resistance development. Normal human microbiota act as a barrier against colonisation by potentially pathogenic microorganisms or overgrowth of present organisms: this is known as "colonisation resistance". Antimicrobials can detrimentally affect this microbiota, first by causing resistance, which can then be transferred by resistance genes to other microorganisms, and secondly by damaging colonisation resistance and allowing colonisation by pathogenic microorganisms or overgrowth of present microorganisms, which may be resistant. The degree of disturbance an antimicrobial may cause to microbiota depends on its spectrum, route of administration, degree of absorption, route of elimination, and inactivation either enzymatically or by binding. Using antimicrobials that cause minimal disturbance to the normal human microbiota reduces the risk of emergence and spread of resistance strains between patients and the transfer of resistance genes between microorganisms [47].

Antimicrobials are distributed throughout the body and are present in different tissues (respiratory tract, urinary tract, digestive tract) at differing concentrations. It is difficult to measure the concentrations of drugs at different sites simultaneously so in vitro models have tried to estimate this [48-50]. Animal models are also used to look at tissue distribution in major organs (lungs, spleen, kidneys, intestines, etc.) and data often correlates to explain efficacy at site of infection or guide the selection of formulations to improve drug disposition. The microbiota present in these sites are an important source of resistance development, especially as the bacteria in these locations are usually present in large numbers and the antimicrobials may be low in concentration. Low concentrations of antimicrobials are often associated with the development of resistance $[33,51,52]$.

Oh et al. investigated the effect of the administration of clindafloxacin (a broad-spectrum quinolone) on intestinal microbiota in 12 human volunteers, who could be considered to be antibiotic naïve [53]. Oral administration resulted in high antimicrobial levels in the faeces of patients, and there was significant emergence of clindafloxacin resistant Gram-negative bacilli. As a result of their findings, Oh et al. suggested the restricted use of clindafloxacin. A comparison of resistance development to antimicrobials that are mainly excreted in bile, such as clindafloxacin, with those which are mainly 
renally excreted, such as amoxicillin, has been made and it has been suggested that there is more resistance development against those which are excreted in bile due to the affect on the microbiota in the large bowel [54]. This is an important area for future research that could inform future drug development and use of current antimicrobials. Rashid et al. compiled data on the ecological impact of new antimicrobial agents (ceftobiprole, ceftarolone, telavancin, dalbavancin, and tigecyclin) on the gastrointestinal microbiota of healthy volunteers and found them to have only minor ecological effects [47]. Ertapenem is primarily renally excreted and was expected to have a small affect on gut microbiota. However, a study of 10 healthy volunteers where ceftriaxone and ertapenem were administered intravenously once daily at dosages $1 \mathrm{~g}$ and $2 \mathrm{~g}$ respectively found considerable ertapenem in faeces and its impact on gut microbiota was similar to that of ceftriaxone [55]. A clinical trial of adults with complicated intra-abdominal infections, treated with piperacillin-tazobactam $(3.375 \mathrm{~g}$ every 6 hours), or ertapenem ( $1 \mathrm{~g}$ once a day) for 4 to 14 days, were compared for bowel colonisation with resistant bacteria. Lower acquisition of resistant Enterobacteriaceae was found with ertrapenem [56].

Orally administered sustained-release formulations can be used to reduce the frequency of dosing required for antimicrobials with relatively short half-lives [57]. Orally administered antimicrobials are predominantly excreted in bile and sustained-release formulations result in an even higher level of drug in the colon and a greater potential for resistance to develop. The effect of orally administered sustained-release delivery systems on the development of resistance in the microbiota of rats has been studied and it was found that oral sustained-release amoxicillin resulted in more resistance [58].

Goldstein reviewed the effects of antimicrobials on gut microbiota in the hospital environment [59]. As opposed to the healthy volunteers in the studies conducted by Oh et al., the patients discussed here could be considered to be antibiotic non-naïve. Clostridium difficle infection was highlighted as a growing problem in hospitals worldwide, particularly due to the disruption of gut microbiota that occurs with the use of broad-spectrum antimicrobials. Infection control measures and antimicrobial stewardship were outlined as two important strategies for Clostridium difficle infection prevention. Another important example discussed was the risk of intestinal colonization with resistant bacteria in patients receiving antimicrobials for intra-abdominal infections. Optimizing Intra-Abdominal Surgery with Invanz studies (OASIS-I and OASIS-II) suggest that resistant Enterobacteriaceae is less like to develop in patients treated with ertapenem compared to piperacillin/tazobactam. Furthermore, recent studies suggest the increased use of non-pseudomonal carbapenems (for example, ertapenem) does not decrease the susceptibility of Pseudomonas aeruginosa to the pseudomonal carbapenems (such as imipenem and meropenem). Indeed, increased ertapenem and decreased imipenem use in one study showed increased susceptibility of Pseudomonas aeruginosa to imipenem.

Examples of resistance gene transfer during therapy involving microbiota organisms have been identified. They include the isolation of Klebsiella pneumoniae carbapenemase (KPC) 3-producing Escherichia coli from a carrier of KPC-3-producing Klebsiella pneumonia, where the patient's gut flora contained a carbapenem-susceptible Escherichia coli strain isogenic with the KPC-3-producing isolate, suggesting horizontal interspecies plasmid transfer [60]. Also the isolation of Staphylococcus epidermis and methicillin-resistant Staphylococcus aureus, both expressing a plasmid expressing resistance gene mupA, suggests a transfer of resistance gene mupA from Staphylococcus epidermis to methicillin-resistant Staphylococcus aureus during a trial of mupirocin for nasal decolonization [61]. 
A greater understanding of microbiota in the body is required to understand the true impact of antimicrobials on microbiota and the generation of resistance. Much is known about the oral microbiota, with around 700 species identified [62]. However, only 50\% of the organisms are culturable so techniques such as pyro-sequencing analysis are being used to study the oral microbiota in more detail [63]. Culture-independent techniques have also enhanced the understanding of microbiotal communities in the colon [64]. Knowledge about skin microbiota is currently mostly from culture based studies with only a few culture-independent studies reported $[65,66]$. Culture-independent approaches have an important role to play in the understanding of human microbiota, which is necessary to increase our understanding of the development of resistance there.

\subsection{Combination Therapy}

The use of combination therapy is common in clinical practice for a number of reasons. First, preventing antimicrobial resistance. The regimen for which the use of combination therapy to prevent bacterial resistance is best established is the treatment of Mycobacterium tuberculosis. The British Medical Research Council, led by Denis Mitchison and Wallace Fox, pioneered multi-drug regimens for tuberculosis using the first randomised clinical trials [67,68]. This concept was extended to treatment of leprosy, human immunodeficiency virus, and cancer. Combinations are used in these cases because resistance to agents develops relatively easily. Such combinations have been most successful, since Mycobacterium tuberculosis develops resistance by chromosomal mutations, rather than by plasmid transfer, and since the organism lives on its own in the tissues, and does not rely of the transfer of resistance from other bacterial species. Other examples of combinations that are used to delay resistance development to the individual components are co-trimoxazole (sulphonamide and trimethoprim); lincomycine and spectinomycine; and aminoglycosides and colistin [69].

Second, some antimicrobials enhance one another's anti-bacterial activity: in other words are synergistic. An example of such a combination is the use of penicillin and gentamicin for bacterial endocarditis.

Third, when a critically ill patient is admitted with suspected septicaemia, and the pathogen is unknown at that stage, doctors treat with several antimicrobials in order to broaden the spectrum of species of bacteria that are targeted [70]. For instance, a combination of a beta-lactam and an aminoglycoside may be used.

Fourth, combinations can specifically target resistant bacteria. For example, the addition of clavulanic acid, which is a beta-lactamase inhibitor, to amoxicillin, renders the amoxicillin active against some beta-lactamase producing bacteria. For instance, Dagan et al. compared amoxicillin with amoxicillin plus clavulanic acid (Augmentin) for the treatment of non-bullous impetigo [71]. Staphylococcus aureus was isolated from all patients and it was found that all isolates of Staphylococcus were sensitive to Augmentin but resistant to amoxicillin. The clinical response was significantly better in the Augmentin recipients. Bacteria produce many different kinds of beta-lactamases and so, potentially, this is an on-going approach for the enhancement of old antibiotics. For example, recent in vivo studies in mice show efficacy of meropenem/clavulanate and amoxicillin/clavulanate combinations against replicating and non-replicating Mycobacterium tuberculosis [72]. 
Fifth, if dormant bacteria are important in an infection. For example, the addition of anti-dormancy agents such as pyrazinamide and rifampicin to combinations for the treatment of tuberculosis has shortened the duration of chemotherapy from 18 to 6 months [38].

A meta-analysis (including data from eight randomised controlled trials) that compared aminoglycoside/beta-lactam combination therapy with beta-lactam mono-therapy to observe the emergence of antimicrobial resistance found that aminoglycoside/beta-lactam combination therapy was not associated with reduced development of resistance when compared with beta-lactam therapy alone [73].

Animal model studies in the 1980s suggested that the addition of an aminoglycoside to beta-lactam therapy might prevent the development of resistance, particularly in the treatment of Pseudomonas aeruginosa [74,75]. Human trials in patients with cystic fibrosis also showed that Pseudomonas aeruginosa resistance occurred less frequently during combination compared to single therapy [76]. The high frequency of chromosomal mutations in Pseudomonas spp. was suggested to support the role of combination therapy [75]. As well as prevention of resistance, suggested benefits of combination therapy for Pseudomonas aeruginosa include antimicrobial synergy and improved adequacy of empirical therapy. The benefits of combination therapy must be balanced against the risk of creating multi-drug resistance organisms [77]. This may be of particular concern if the effect of agents on bacteria, initially not involved in the pathogenesis, results in horizontal gene transfer of resistance genes to the pathogens.

Combination therapy for the treatment of Neisseria gonorrhoeae has recently been suggested as potentially reducing the development of antimicrobial resistance but we could find no controlled study data on this [78]. Bonhoeffer et al. used a mathematical model to assess whether combination therapy might be useful in the treatment of Neisseria gonorrhoeae and concluded that resistance might be preventable with more widespread use of combination therapy [79].

\subsection{Choice of Antimicrobial}

\subsubsection{Bacteria-Antimicrobial Pairings}

The rates of spontaneous resistance mutations for bacteria-antimicrobial pairings at certain antimicrobial concentrations are known. By considering the bacterial loads in different infected lesions, it is possible to estimate whether or not resistance to an antimicrobial is likely to develop. In some clinical situations, the probability of resistant variants being selected is so high that certain antimicrobials may be best avoided [42]. Bacteria-antimicrobial pairings that are considered high risk for the development of resistance due to de novo mutations include rifampicin and all species of bacteria; quinolones and Staphylococcus aureus or Pseudomonas aeruginosa; and cephalosporins and Enterobacter spp. or Citrobacter spp [26]. In contrast to rifampicin, for example, it has been suggested that antimicrobials that target the cell membrane may induce less resistance [80]. Multi-targeting versus single-target antimicrobials is another exciting area of investigation [81].

\subsubsection{Rapid Diagnostic Techniques}

Narrow-spectrum antimicrobials should be used instead of broad-spectrum antimicrobials where possible to avoid affecting microbiota. Use of more narrow-spectrum antimicrobials may reduce 
incidence of Clostridium difficile infection. Culturing bacteria can take days; therefore non-targeted broad-spectrum antimicrobials are often used first before sensitivities are known. Rapid diagnostic tests have been developed, which can help doctors target bacteria more quickly [5]. Group A betahaemolytic streptococcus (GABHS) is the most common bacterial cause of pharyngitis [82]. Throat swab culture is the gold standard for diagnosis but results take one to two days. Rapid antigen detection testing gives results in 10-15 minutes (specificity greater than or equal to $95 \%$, sensitivity greater than or equal to 90\%). Madurell et al. showed that the use of rapid antigen detection tests to identify GABHS in patients with acute pharyngitis helped doctors identify target bacteria and devise a rational treatment approach. Procalcitonin (PCT) can be used as a biomarker for bacterial infections [83]. Burkhardt et al. compared PCT-guided antimicrobial treatment to standard care in patients with an acute respiratory tract infection. They showed that PCT-guided treatment reduced the antimicrobial treatment rate by $41.6 \%$ [84].

\subsubsection{Antibiotic Surveillance and Stewardship}

The choice of antimicrobials is generally based on guidelines, which include knowledge of surveillance of bacterial susceptibility, and resistance to antimicrobials in different regions and countries. Targeted programs of active surveillance can potentially be used to guide empirical therapy [85]. Different types of surveillance used include governmental, institutional, industrial, and pharmaceutical programs, but there will inevitably be a time-lag between the results of surveillance systems and decision making in clinical contexts [86]. Antibiotic stewardship is coordinated interventions that aim to measure and improve antimicrobial usage. This includes the promotion of appropriate selection of antimicrobials, dosage, route, and duration of therapy.

\subsubsection{Antimicrobial Cycling}

Antimicrobial cycling is scheduled rotations of antimicrobial usage between drugs with a similar spectrum of activity. The principle is that the more often an antimicrobial is prescribed, the more resistance will develop against it, and withdrawing antimicrobials for a time will remove the selective pressure, allowing resistance rates to stabilize or fall. It is important to note that the restriction of sulphonamide use nationally in the UK did correlate with a reduction in the resistance of sulphonamide-resistant Escherichia coli [87]. Bennett et al. reported an improvement in the antimicrobial susceptibility profile of Gram-negative organisms (Pseudomonas aeruginosa, Escherichia coli, and Klebsiella pneumoniae) in a surgical intensive care unit compared to the medical intensive unit after the implementation of rotation of piperacillin/tazobactam, imipenem/cilastin, ceftazidime, and ciprofloxacin as the primary antibiotic used to treat suspected gram-negative infections every month [88]. However, a review of both clinical trials and mathematical modelling studies examined the efficacy of antimicrobial cycling to prevent resistance and reported there was not evidence to support the implementation of antimicrobial cycling as a strategy for reducing antimicrobial resistance in hospitals [89]. 


\section{Conclusions}

Three core strategies to extend the lifespan of our current antimicrobials are: (i) reducing usage of antimicrobials; (ii) better use of antimicrobials; and (iii) infection control. This review examines the literature relating to the second strategy, to use antimicrobials more appropriately, concentrating on three areas: pharmacokinetics/pharmacodynamics, combination therapy, and appropriate choice of antimicrobials.

For mycobacterium tuberculosis the use of multi-drug combination therapy is an established strategy being used to prevent resistance to treatment. Furthermore, it has recently been suggested that the efficacy of the core anti-tuberculosis drugs, isoniazid and rifamycins, is determined by peak drug concentrations. This may mean that tuberculosis regimes can be shortened and, if so, an improvement in adherence would hopefully mean a reduction in resistance to treatment.

For other bacterial infections, strategies to use antimicrobials in a way that will reduce resistance development are yet to be clearly established. We hope that the subject areas we have outlined in this article can be developed to help us to use our existing antimicrobials in ways that will extend their lifespan by preventing antimicrobial resistance.

\section{Conflict of Interest}

The authors declare no conflict of interest.

\section{References}

1. Boucher, H.; Talbot, G.H.; Bradley, J.S. Bad bugs, no drugs: no ESKAPE! An Update from the Infectious Diseases Society of America. Clin. Infect. Dis. 2009, 425, 1-12.

2. Rice, L.B. Federal funding for the study of antimicrobial resistance in nosocomial pathogens: no ESKAPE. J. Infect. Dis. 2008, 197, 1079-1081.

3. Arias, C.A.; Murray, B.E. Antibiotic-resistant bugs in the 21 st century - a clinical super challenge. N. Engl. J. Med. 2012, 360, 439-443.

4. Livermore, D.M. Has the era of unbeatable infections arrived? J. Antimicrob. Chemother. 2009, 64, 29-36.

5. Carlet, J.; Jarlier, V.; et al. Ready for a world without antibiotics? The Pensieres Antibiotic Resistance Call to Action. Antimicrob. Resist. Infect. Contr. 2012, 1, 11.

6. Landman, D.; Georgescu, C.; Martin, D.A.; Quale, J. Polymyxins Revisited. Clin. Microbiol. Rev. 2008, 21, 449-465.

7. Coates, A.R.M.; Halls, G.; Hu, Y. Novel classes of antibiotics or more of the same? Brit. J. Pharmacol. 2009, 163, 184-194.

8. Talbot, G.H.; Bradley, J.; Edwards, J.E., Jr; Gilbert, D.; Scheld, M.; Bartlett, J.G. Bad bugs need drugs: an update on the development pipeline from the antimicrobial availability task force of the Infectious Diseases Society of America. Clin. Infect. Dis. 2006, 42, 657-668.

9. Talbot, G.H. What is the pipeline for Gram-negative pathogens? Expert Rev. Anti. Infect. Ther. 2008, 6, 39-49. 
10. Laudano, J.B. Ceftaroline fosamil: a new broad-spectrum cephalosporin. J. Antimicrob. Chemother. 2011, 66, 11-18.

11. Lopez-Rojas, R.; Sanchez-Cespedes, J.; Docobo-Perez, F.; Domınguez-Herrera, J.; Vila, J.; Pachon, J. Pre-clinical studies of a new quinolone (UB-8902) against Acinetobacter baumannii resistant to ciprofloxacin. Int. J. Antimicrob. Agents. 2011, 38, 355-359.

12. Sutcliffe, J.A. Antibiotics in development targeting protein synthesis. Ann. N. Y. Acad. Sci. 2011, $1241,122-152$.

13. Caron, W.; Mousa, S. Prevention strategies for antimicrobial resistance: a systematic review of the literature. Infection and Drug Resistance. 2010, 3, 25-33.

14. Hughes, J.M. Preserving the lifesaving power of antimicrobial agents. JAMA. 2011, 305, 1027-1028.

15. Rashmi, S. Antibacterial resistance: Current problems and possible solutions. Indian J. Med. Sci. 2005, 59, 120-129.

16. World Health Organization. Global Strategy for Containment of Antimicrobial Resistance. World Health Organization 2001. Available online at: http://www.who.int/csr/resources/publications/ drugresist/WHO_CDS_CSR_DRS_2001_2_EN/en/ (access on 6 June 2013).

17. Sharma, R.; Sharma, C.L.; Kapoor, B. Antibacterial resistance: Current problems and possible solutions. IJMS 2005, 59, 120-129.

18. Huttner, B.; Goossens, H.; Verheji, T.; Harbath, S. Characteristics and outcomes of public campaigns aimed at improving the use of antibiotics in outpatients in high-income countries. Lancet 2010, 10, 17-31.

19. Gould, I.M. A review of the role of antibiotic policies in the control of antibiotic resistance. JAC 1999, 43, 459-465.

20. Collignon, P.J. Antibiotic resistance. Med. J. Aust. 2002, 177, 325-329.

21. Molstad, S.; Erntell, M.; Hanberger, H.; et al. Sustained reduction of antibiotic use and low bacterial resistance: 10-year follow-up of the Swedish Strama programme. Lancet Infect. Dis. 2008, $8,125-132$.

22. Nicole, L.E. Infection control programmes to contain antimicrobial resistance. WHO, Department of Communicable Disease Surveillance and Response, 2001. Available online at: http://www.who.int/csr/resources/publications/drugresist/WHO_CDS_CSR_DRS_2001_7/en/ (access on 6 June 2013).

23. Essack, S.Y. Strategies for the Prevention and Containment of Antibiotic Resistance. S. A. Fam. Pract. 2006, 48, 51a-51d.

24. Drlica, C. The mutant selection window and antimicrobial resistance. JAC 2003, 52, 11-17.

25. Vaidya, V. K. Horizontal Transfer of Antimicrobial Resistance by Extended-Spectrum BetaLactamase-Producing Enterobacteriaceae. J. Lab. Physicians. 2011, 3, 37-42

26. Barker, K. Antibiotic resistance: a current perspective. Br. J. Clin. Pharmacology. 1999, 48, 109-124.

27. Olofsson, S.K.; Cars, O. Optimizing drug exposure to minimize selection of antibiotic resistance. Clin. Infect. Dis. 2007, 1, S129-S136.

28. Blaser, J.; Stone, B.B.; Griner, M.C.; Zinner, S.H. Comparative study with enoxacin and netilmicin in a pharmacodynamics model to determine importance of ratio of antibiotic peak concentration to MIC for bactericidal activity and emergence of resistance. Antimicrob. Agents Chemother. 1987, 31, 1054-1060. 
29. Marchbanks, C.R.; McKiel, J.R.; Gilbert, D.H.; et al. Dose ranging and fractionation of intravenous ciprofloxacin againsts Pseudomonas aruginosa and Staphylococcus aureus in an in vitro model of infection. Antimicrob. Agents Chemother. 1993, 37, 1756-1763.

30. Drusano, G.I.; Johnson, D.E.; Rosen, M.; Standiford, H.C. Pharmacodynamics of a fluroquinolone antimicrobial agent in a neutropenic rat model of Pseudomonas species. Antimicrob. Agents Chemother. 1993, 37, 483-490.

31. Stearne, L.E.; van Boxtel, D.; Lemmens, N.; Goessens, W.H.; Mouton, J.W.; Gyssens, I.C. Comparative study of the effects of ceftizoxime, piperacillin, and piperacillin-tazobactam concentrations on antibacterial activity and selection of antibiotic-resistant mutants of Enterobacter cloacae and Bacteroides fragilis in vitro and in vivo in mixed-infection abscesses. Antimicrob. Agents Chemother. 2004, 48, 1688-1698.

32. Wiuff, C.; Lykkesfeldt, J.; Svendsen, O.; Aarestrup, F.M. The effects of oral and intramuscular administration and dose escalation of enrofloxacin on the selection of quinolone resistance among Salmonella and coliforms in pigs. Res. Vet. Sci. 2003, 75, 185-193.

33. Guillemot, D.; Carbon, C.; Balkau, B.; et al. Low dosage and long treatment duration of b-lactam: risk factors for carriage of penicillin-resistant Streptococcus pneumoniae. JAMA 1998, 279, 365-370.

34. Davidson, R.; Cavalcanti, R.; Brunton, J.L.; et al. Resistance to levofloxacin and failure of treatment of pneumococcal pneumonia. N. Engl. J. Med. 2002, 346, 747-750.

35. Thomas, J.K.; Forrest, A.; Bhavnani, S.M.; et al. Pharmacodynamic evaluation of factors associated with the development of bacterial resistance in acutely ill patients during therapy. Antimicrob. Agents Chemother. 1998, 42, 521-527.

36. Mitchison, D. Pharmacokinetic/pharmacodynamic parameters and the choice of high-dosage rifamycins. Int. J. Tuberc. Lung Dis. 2012, 16, 1186-1189.

37. Mitchison, D. The diagnosis and therapy of tuberculosis during the past 100 years. Am. J. Respir. Crit. Care Med. 2005, 171, 699-706.

38. Mitchison, D. How drug resistance emerges as a result of poor compliance during short course chemotherapy for tuberculosis. Int. J. Tuberc. Lung Dis. 1998, 2, 10-15.

39. Blondeau, J.M.; Zhao, X.; Hansen, G.; Drilica, K. Mutant Prevention Concentrations of Fluoroquinolones for Clinical Isolates of Streptococcus pneumoniae. Antimicrob. Chemother. 2001, 45, 433-438.

40. Cui, J.; Liu, Y.; Wang, R.; Tong, W. Drlica, K.; Zhao, X. The mutant selection window in rabbits infected with Staphylococcus aureus. J. Infect. Dis. 2006. 194, 1601-1608.

41. Zhu Y.L.; Hu, L.F.; Mei, Q.; Cheng, J.; Liu, Y.; Y.; Ye, Y.; Li, J.B. Testing the mutant selection window in rabbits infected with methicillin-resistant Staphylococcus aureus exposed to vancomycin. J. Antimicrob. Chemother. 2012, 67, 2700-2706.

42. Williams, J.; Sefton, A. The Prevention of Antibiotic Resistance during Treatment. Infection 1999, 27, 29-31.

43. Lee, M. Lee, J.; Carroll, M.W.; et al. Linezolid for treatment of chronic extensively drug-resistant tuberculosis. N. Engl. J. Med. 2012, 367, 1508-1518.

44. Craig, W.A. Does the dose matter? Clin. Infect. Dis. 2001, 15, S233-S237.

45. Drusano, G.L. Prevention of resistance: a goal for dose selection for antimicrobial agents. Clin. Infect. Dis. 2003, 36, S42-S50. 
46. Geli, P.; Laxminarayan, R.; Dunne, M.; Smith, D.L. ' One-Size-Fits-All’’? Optimizing Treatment Duration for Bacterial Infections. PLOS one 2012, 7, 1-10.

47. Rashid, M.U.; Weintraub, A.; Nord, C.E. Effect of new antimicrobial agents on the ecological balance of human microflora. Anaerobe 2012, 18, 249-253.

48. Brunner, M.; Derendorf, H.; Muller, M. Microdialysis for in vivo pharmacokinetic/pharmacodynamic characterization of anti-infective drugs. Curr. Opin. Pharmacol. 2005, 5, 495-499.

49. Cars, O. Pharmacokinetics of antibiotics in tissues and tissue fluids: a review. Scand. J. Infect. Dis. Suppl. 1990, 74, 23-33.

50. Cars, O.; Ogren, S. Antibiotic tissue concentrations: methodological aspects and interpretation of results. Scand. J. Infect. Dis. Suppl. 1985, 44, 7-15.

51. Gullberg, E.; Cao, S.; Berg, O.G.; Ilbäck, C.; Sandegren, L.; Hughes, D. Selection of Resistant Bacteria at Very Low Antibiotic Concentrations. PLoS Pathog. 2011, 7, e1002158.

52. Zhao, X.; Drlica, K. Restricting the Selection of Antibiotic-Resistant Mutants: A General Strategy Derived from Fluoroquinolone Studies. Clin. Infect. Dis. 2001, 333, S147-S156.

53. Oh, H.; Nord, C.E.; Barkholt, L.; Hedberg, M.; Edlund, C. Ecological disturbances in intestinal microflora caused by clinafloxacin, an extended- spectrum quinolone. Infection. 2000, 28, 272-277.

54. Lode, H.; Von der Höh, N.; Ziege, S.; Borner, K.; Nord, C.E. Ecological effects of linezolid versus amoxicillin/clavulanic acid on the normal intestinal microflora. Scand. J. Infect. Dis. 2001, 33, 899-903.

55. Pletz, M.W.R.; Rau, M.; Bulitta, J.; De Roux, A.; et al. Ertapenem Pharmacokinetics and Impact on Intestinal Microflora, in Comparison to Those of Ceftriaxone, after Multiple Dosing in Male and Female Volunteers. Antimicrob. Agents Chemother. 2004, 48, 3765-3772.

56. DiNubile, M. J.; Chow, J. W.; Satishchandran, V.; Polis, A.; Motyl, M. R.; Abramson, M. A.; Teppler, H. Acquisition of Resistant Bowel Flora during a Double-Blind Randomized Clinical Trial of Ertapenem versus Piperacillin-Tazobactam Therapy for Intraabdominal Infections. Antimicrob. Agents Chemother. 2005, 49, 3217-3221.

57. Isha, C.; Nimrata, S.; Rana, A.C.; Surbhi, G. Oral sustained release drug delivery system: an overview. Int. Res. J. Pharm. 2012, 3, 57-62.

58. Hoffman, A.; Horwitz, E.; Hess, S.; et al. Implications on emergence of antimicrobial resistance as a critical aspect in the design of oral sustained release delivery systems of antimicrobials. Pharm. Res. 2008, 25, 667-671.

59. Goren, M.G.; Carmeli, Y.; Schwaber, M.J.; Chmelnitsky, I.; Schechner, V.; Navon-Venezia, S.Transfer of Carbapenem-Resistant Plasmid from Klebsiella pneumoniae ST258 to Escherichia coli in Patient. Emerg. Infect. Dis. 2010, 16, 1014-1017.

60. Goldstein, E.J. Beyond the target pathogen: ecological effects of the hospital formulatory. Curr. Opin. Infect. Dis. 2011, 1, S21-S31.

61. Hurdle J.G.; O’Neill, A.J.; Mody, L.; Chopra, I.; Bradley, S.F. In vivo transfer of high-level mupirocin resistance from Staphylococcus epidermidis to methicillin-resistant Staphylococcus aureus associated with failure of mupirocin prophylaxis. J. Antimicrob. Chemother. 2005, 56, 1166-1168.

62. Paster, B.J.; Olsen, I.; Aas, J.A.; Dewhirst, F.E. The breadth of bacterial diversity in the human periodontal pocket and other oral sites. Periodontol 2000 2006, 42, 80-87. 
63. Keijser, B.J.F.; Zaura, E.; Huse, S.M.; van der Vossen, J.M.B.M.; Schuren, F.H.J.; Montijn, R.C.; ten Cate, J.M.; Crielaard, W. Pyrosequencing analysis of the oral microflora of healthy adults. J. Dent. Res. 2008, 87, 1016-1020.

64. Gill, S.R.; Pop, M.; Deboy, R.T.; Eckburg, P.B.; Turnbaugh, P.J.; Samuel, B.S.; Gordon, J.I.; Relman, D.A.; Fraser-Liggett, C.M.; Nelson, K.E. Metagenomic analysis of the human distal gut microbiome. Science 2006, 312, 1355-1359.

65. Gao, Z.; Tseng, C.H.; Pei, Z.; Blaser, M.J. Molecular analysis of human forearm superficial skin bacterial biota. Proc. Natl. Acad. Sci. USA 2007, 104, 2927-2932.

66. Fierer, N.; Hamady, M.; Lauber, C.L.; Knight, R. The influence of sex, handedness, and washing on the diversity of hand surface bacteria. Proc. Natl. Acad. Sci. USA. 2008, doi: 10.1073/pnas.0807920105.

67. Mitchison, D. Problems of drug resistance. Br. Med. Bull. 1954, 69, 640-641.

68. Fox, W.; Sutherland, I.; Daniels, M. A five-year assessment of patients in a controlled trial of streptomycin in pulmonary tuberculosis. Q. J. Med. 1954, 23, 347-366.

69. Grüneberg, R.N. The microbiological rationale for the combination of sulphonamides with trimethoprim. J. Antimicrob. Chemother. 1979, 5, 27-36.

70. Boyd, N.; Nailor, M.D. Combination antibiotic therapy for empiric and definitive treatment of gram-negative infections: insights from the Society of Infectious Diseases Pharmacists. Pharmacotherapy 2011, 31, 1073-1084.

71. Dagan, R.; Bar-David, Y. Comparison of amoxicillin and clavulanic acid (augmentin) for the treatment of nonbullous impetigo. Am. J. Dis. Child. 1989, 143, 916-918.

72. Solapure, S.; Dinesh, N.; Shandil, R.; Ramachandran, S. S.; et al. In vitro and in vivo efficacy of beta-lactams against replicating and slowly growing/non replicating M. tuberculosis. Antimicro. Agents Chemo. 2013, 57, 2506-2510.

73. Bliziotis, I.; Samonis, G.; Vardakas, K.; Chrysanthopoulou, S.; Falagas, M. Effect of Aminoglycoside and b-Lactam Combination Therapy versus b-Lactam Monotherapy on the Emergence of Antimicrobial Resistance: A Meta-analysis of Randomized, Controlled Trials. Clin. Infect. Dis. 2005, 41, 149-158.

74. Gerber, A.U.; Vastola, A.P.; Brandel, J.; Craig, W.A. Selection of aminoglycoside-resistant variants of Pseudomonas aeruginosa in an in vivo model. J. Infect. Dis. 1982, 146, 691-697.

75. Mouton, J. Combination Therapy as a Tool to Prevent Emergence of Bacterial Resistance. Infection. 1999, 27, 24-28.

76. Michalsen, H.; Bergan, I. Azlocillin with and without an aminoglycoside against respiratory tract infections in children with cystic fibrosis. Scand. J. Infect. Dis. 1981, 29, 92-97.

77. Traugott, K.A.; Echevarria, K.; Maxwell, P.; Green, K.; Lewis, J.S., $2^{\text {nd }}$. Monotherapy or combination therapy? The Pseudomonas aeruginosa conundrum. Pharmacotherapy. 2011, 31, 598-608.

78. Unemo, M.; Shafer, W.M. Antibiotic resistance in Neisseria gonorrhoeae: origin, evolution, and lessons learned for the future. Ann. N. Y. Acad. Sci. 2011, 1230, E19-E28.

79. Bonhoeffer, S.; Lipsitch, M.; Levin, B.R. Evaluating treatment protocols to prevent antibiotic resistance. Proc. Natl. Acad. Sci. USA 1997, 94, 12106-12111. 
80. Hurdle, J.; O’Neill, A.; Chopra, I.; Lee, R. Targeting bacterial membrane function: an unexploited mechanism for treating persistent infections. Nat. Rev. 2011, 9, 62-75.

81. Silver, L.L. Multi-targeting by monotherapeutic antibacterials. Nat. Rev. Drug Discov. 2007, 6, 41-55.

82. Leung, A.K.; Newman, R.; Kemar, A.; Davies, D.H. Rapid antigen detection testing in diagnosing group A beta-hemolytic streptococcal pharyngitis. Expert Rev. Mol. Diagn. 2006, 6, 761-766.

83. Madurell, J.; Balagué, M.; Gomez, M.; Cots, J.M.; Llor, C. Impact of rapid antigen detection testing on antibiotic prescription in acute pharyngitis in adults. Faringocat Study: a multicentric randomized controlled trial. BMC Fam. Pract. 2010, 11, 25-29.

84. Burkhardt, O.; Ewig, S.; Giesdorf, S.; Giersdorf, S.; Harmann, O.; Wegscheider, K.; HummersPradier, E.; Welte, T. Procalcotonin guidance and reduction of antibiotic use in acute respiratory tract infection. Eur. Respir. J. 2010, 36, 601-607.

85. Harris, A.D.; Furuno, J. P.; et al. Targeted Surveillance of Methicillin-Resistant Staphylococcus aureus and Its Potential Use to Guide Empiric Antibiotic Therapy. Antimicrob. Chemother. 2010, 54, 3143-3148.

86. Masterton, R.G. Surveillance studies: how can they help the management of infection? JAC. 2000, $46,53-58$.

87. Enne, V.I.; Livermore, D.M.; Stephens, P.; Hall, L.M.C. Persistence of sulphonamide resistance in Escherichia coli in the UK despite national prescribing restriction. Lancet 2001, 357, 1325-1328.

88. Bennett, K.M.; Scarborough, J.E.; Sharpe, M.; et al. Implementation of antibiotic rotation protocol improves antibiotic susceptibility profile in a surgical intensive care unit. J. Trauma. 2007, 63, 307-311.

89. Brown, E.; Nathwani, D. Antibiotic cycling: a systematic review of the evidence of efficacy. JAC. 2005, 55, 6-9.

(C) 2013 by the authors; licensee MDPI, Basel, Switzerland. This article is an open access article distributed under the terms and conditions of the Creative Commons Attribution license (http://creativecommons.org/licenses/by/3.0/). 